\title{
Age influences magnitude but not duration of response to levodopa
}

\author{
Raymon Durso, Kathryn Isaac, Linda Perry, Marie Saint-Hilaire, Robert G Feldman
}

\begin{abstract}
Following an all-night fast, 45 patients with Parkinson's disease were examined using certain motor items present in the United Parkinson's Disease Rating Scale. All were given a single tablet of carbidopa $25 \mathrm{mg}$ and levodopa $250 \mathrm{mg}$ and re-examined 90 minutes later. In addition to this evaluation, 23 of these patients underwent further scoring over a 4-hour period. A significant negative correlation was found between age and one important aspect of drug-derived benefit: magnitude of response. In contrast, age had no apparent influence on duration of benefit from the drug. Although baseline (fasting) scores were predictably correlated with duration of disease, magnitude of response was not adversely influenced by this variable. Not all Parkinsonian signs were equally influenced by age. Whereas the poor response of gait and bradykinesia appeared to be dependent on age, no such effect was noted on rest tremor scores. The data indicate that in patients with Parkinson's disease treated long term, factors associated with age rather than duration of disease may have a stronger adverse influence on magnitude of response to levodopa.
\end{abstract}

$(\mathcal{F}$ Neurol Neurosurg Psychiatry 1993;56:65-68)

A well-documented finding regarding limitations of levodopa use in Parkinson's disease is the observation that treatment is most efficacious during the early years of therapy and that, later, many patients experience a significant decline in the effectiveness of the drug. ${ }^{2}$ Two factors that characterise this decline are, firstly, a shorter duration of response and, secondly, an inability to maintain the same quality of improvement. The reduction in the duration of response to levodopa is clinically manifested as wearing off, end-of-dose deterioration or the on-off phenomenon. There is considerable agreement that these limitations in duration of benefit are, at least in part, related to disease progression (that is, a continuing loss of cells in the nigra)..$^{3-6}$ In this regard, a diminished capacity of nigral terminals to synthesize and store dopamine, or both, ${ }^{78}$ may play an important role. There is less consensus about factors that may limit the magnitude of the response to levodopa. Using data obtained from patients participating in pharmacological studies at the
Boston Veterans Administration Medical Center and University Hospital, we analysed the duration and magnitude of response to levodopa in relation to several potential factors that might influence its efficacy. This opportunity was available because all participants had received the same dose of carbidopa and levodopa without concomitant administration of other medications and all had been scored using motor items present in the United Parkinson's Disease Rating Scale (UPDRS).

\section{Patients and methods}

Examination scores from 45 patients (39 males and six females) with Parkinson's disease, having participated in either of two pharmacological studies, were analysed with regard to their age at the time of study; their age at time of disease onset; the duration of the disease and the daily levodopa dose. The duration of levodopa therapy was not specifically addressed as essentially it was identical to the duration of disease in our patient population. The diagnosis of Parkinson's disease had been agreed upon by at least two neurologists experienced in movement disorders and all patients exposed to levodopa had demonstrated sustained, significant benefit from the drug. Individuals with pyramidal, cerebellar or prominent autonomic signs were excluded, to minimise inclusion of the diagnosis of multisystems degeneration. Prior approval for these projects had been given by the Research and Development Human Studies Subcommittees of both the Boston Veterans Administration Medical Center and the Boston University Hospital.

The two pharmacological studies from which subjects were evaluated employed identical methodologies except for frequency of observations following carbidopa and levodopa administration. One patient group (22 patients) was scored immediately preceding administration of carbidopa and levodopa and 90 minutes after. The other group (23 patients underwent multiple examinations immediately before and 30, 60, 90, 120, 180, 240 minutes after administration. No single patient participated in both studies. On the day of investigation, patients were fasted and all medications withheld from midnight. Studies were conducted in both outpatient and inpatient settings. At 9am subjects were evaluated using motor examination items in the UPDRS. These examinations were also videotaped. Scoring was done by either one of two investigators ( $R D$ or $\mathrm{KI})$. A separate analysis of the 
videotape recordings demonstrated that differences between investigators were negligible.

All participants were given a single tablet of carbidopa $25 \mathrm{mg}$ and levodopa $250 \mathrm{mg}$ immediately following the baseline examination and then underwent re-examination according to the predetermined schedule of the pharmcological study. Scores rating resting tremor, rigidity, bradykinesia (opening and closing of the hands) and gait were analysed for magnitude of response in all patients and for duration of response in 23 patients. Tremor and rigidity ratings were based on the average severity present in all four limbs, and bradykinesia scoring represented the average severity present in both hands. Each measured item ranged from zero (no abnormality) to four (marked severity). The magnitude of response was examined in two ways: the percentage change from the baseline (average of all four signs); and the total score (all four signs) 90 minutes after drug administration. The 90 minute time was considered appropriate because the analysis of subjects undergoing multiple examinations revealed the average time from initial drug administration to the onset of maximum improvement (sum of all four signs) to be 96 minutes. In addition, this time did not differ significantly among signs using analysis of variance.

The duration of response (evaluated in 23 patients) was determined by taking the longest time after administration at which druginduced benefit (a reduction in the total score of the four measured signs) could still be noted. This time was then subtracted from the time of the last score immediately before the medication took effect. A ceiling effect was present for some patients; that is, the maximum duration of response that could be recorded was 240 minutes, even for individuals whose benefit extended past the four hour observation period. It should also be noted that the scoring system employed for individual motor items is oriented towards detecting relatively coarse changes in improvement. As a consequence, it is possible that subtle differences indicative of the initial effect of medication could be missed. We believe this source of error to be minimised, however, by the tendency of most chronic patients with Parkinson's disease to experience maximum benefit almost simultaneously with the onset of any clinical response. The latter is frequently evident in patients with previous exposure to levodopa and has been termed the "all or none response". 5

Fasting disease severity (Hoehn and Yahr scale) for all 45 patients ranged from stage 1 to stage 5 with an average score of 3.44 . Their mean age was 63.7 (SD 9, range 40-79) and they averaged 9.3 years from the time of diagnosis to the date of the study (SD 7.2, range 1-37). Of the 45 subjects, 42 were taking carbidopa and levodopa at the time of the study. Their levodopa dose (in the form of Sinemet) averaged $514 \mathrm{mg}$ per day (SD 303, range 150-1500). Two patients had discontinued levodopa for at least two years before the study and one patient had never taken the drug. In addition to carbidopa and levodopa, 16 patients were taking deprenyl, 13 were taking bromocriptine, 10 were on anticholingergic medication, 5 took amantadine and 3 pergolide.

Multiple regression analyses with post hoc tests were used to examine the relationship between response to levodopa and age at the time of study; duration of the disease; and the daily dose of levodopa. In addition, the relative importance of age at the time of study and the age at disease onset with respect to the magnitude of response to levodopa was assessed by multiple regression analysis. Finally, Student's $t$ tests were employed to further analyse the quality and duration of the response to levodopa as a function of age and duration of disease.

\section{Results}

The amplitude of the response to carbidopa and levodopa seemed to be most influenced by age and did not significantly correlate with the duration of disease or previous daily dose of levodopa. Less disability (sum of all four signs) 90 minutes after drug administration was significantly associated with a younger age at the time of study ( $p=0.006)$ but not a shorter duration of disease $(p=0.314)$. The other measure employed to evaluate the magnitude of response to medication, percentage change in total score from the baseline, demonstrated similar results. Specifically, a significant negative correlation $(p=0.0003)$ was found between the percentage change in the total UPDRS score (four signs) at 90 minutes and the age at time of study (figure). No such relationship was noted when comparing percentage change with duration of disease $(p=0.832)$. When patients were separated into groups according to age and duration of disease, $t$ test analyses gave similar results (table).

The percentage response to levodopa as a function of age at the time of the study and age at disease onset was significant for the first $(p=0.020)$ but not the second criterion $(p=0.599)$.

The association of age with a lesser percentage change after drug administration was not evident for all individually measured UPDRS signs. Although amelioration in bradykinesia $(p=0.006)$ and gait $(p=0.002)$ were diminished as a function of age, rest tremor $(p=0.723)$ and rigidity $(p=0.080)$ were not. These observations, relating a poorer response of bradykinesia or gait to levodopa in older compared with younger PD subjects, were independent of baseline scores; that is, there was no significant correlation between age and any individual motor item measured at baseline. In addition, duration of disease or previous daily dose did not correlate significantly with percentage change for any single sign.

We examined the possibility that the better response to levodopa noted in younger patents might be an artefact related to deprenyl medication. Clinical measures (that is, baseline score, 90 minute score or percentage change 


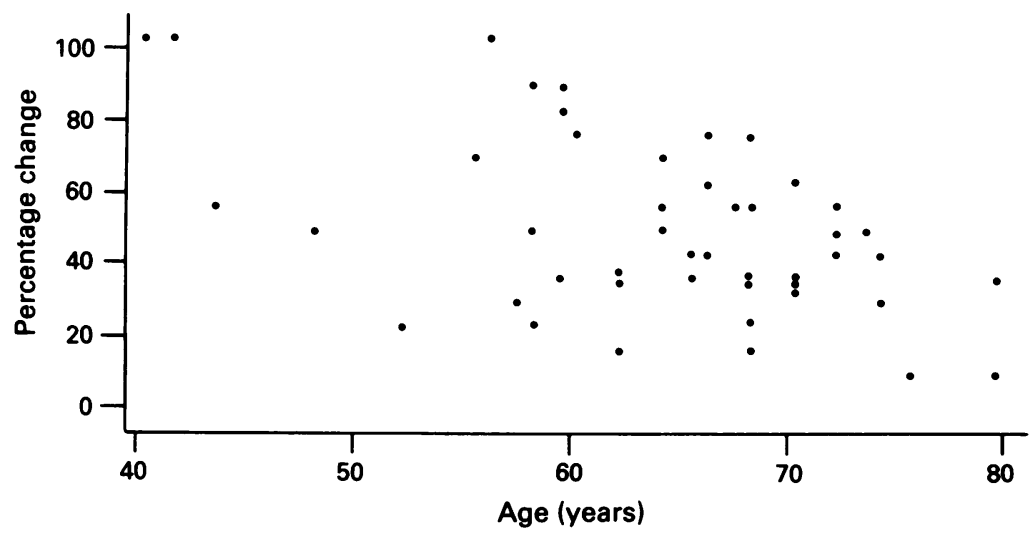

Figure Percentage improvement (average of all four signs) in 45 patients with Parkinson's disease after oral administration of a tablet of carbidopa $25 \mathrm{mg}$ and levodopa $250 \mathrm{mg}$ plotted against patients' ages at the time of the study $(r=-0.537$, $p=0.0003)$. were significantly more likely in younger than older patients regardless of the duration of their disease. This notable influence of age was not observed with baseline scores. There is surprisingly little. in the literature discussing specific effects of age on response to levodopa. In 1972 , Granerus et $a l^{9}$ reported that levodopa related improvement in activities of daily living was negatively correlated to age. More recent studies suggest that patients who develop Parkinsion's disease at a younger age experience a more potent response to levodopa than their older counterparts. ${ }^{10-13}$ For the most part, these latter reports have investigated the hypothesis that young-onset and old-onset Parkinson's disease may be different pathological entities. Our results corroborate an effect of age on efficacy of levodopa and extend previous studies by suggesting that firstly, the principle effect of age is on amplitude rather than duration of response to levodopa; secondly, rest tremor may be a sign that is most resistant to any age-dependent effect and, thirdly, current age rather than age at disease onset may have the greater negative impact on magnitude of response to levodopa.

One mechanism that could mediate the agedependent decline in drug efficacy observed is the loss of striatal receptors. A reduction of dopaminergic binding sites in human basal ganglia correlated with age has been described. ${ }^{1415}$ Similarly, animal models of ageing have demonstrated loss of dopamine receptor binding affinity in striatum ${ }^{16}$ as well as a decline in striatal D1 receptor-related activity (adenylate cyclase ). ${ }^{17}$ Most recently PET, evaluating striatal D2 receptor binding with $\left[{ }^{11} \mathrm{C}\right]$ raclopride in patients with Parkinson's disease, has found decreased activity as a function of age which appears to be independent of exposure to levodopa. ${ }^{19}$ Our results suggest that such an age-related loss might have specific clinical consequences in Parkinson's disease, that is, the limitation of the magnitude of response to levodopa.

Another possible explanation for our agedependent results implicates the substantia nigra. A previous publication from our laboratory has suggested that the magnitude of response to levodopa may be linked to nigral integrity. ${ }^{20}$ Loss of nigral cells as a function of age has been well described. ${ }^{21}$ Furthermore, neuronal subpopulations in the nigra with different susceptibilities to ageing and Parkinson's disease may exist. ${ }^{22}$ It is possible, therefore, that nigral cell loss associated with Parkinson's disease is further compounded by an age-related depletion. This additive ageing effect might limit the response to levodopa by further reducing the capacity of nigrostriatal terminals to adequately convert levodopa to dopamine. $^{2324}$

Finally, an alternative explanation for agedependent findings of levodopa efficacy, as specifically proposed by others, ${ }^{1025}$ is that both young-onset and old-onset Parkinson's disease represents different disease entities. This hypothesis states that patients with the oldonset form suffer more widespread pathology (involving, for example, the striatum and 
neocortex) which limits the effectiveness of levodopa therapy and increases the incidence of other clinical signs such as dementia. We believe this explanation less likely to account for our results as multiple regression analysis using percentage response data indicated a more important role for age at the time of study than the age at disease onset.

We found the tendency for rest tremor response to be least affected by age particularly interesting. This would imply that tremor, compared with other Parkinsonian signs, might remain more responsive to levodopa during long-term therapy (as patients concurrently age). In this regard, both Bonnet et $a l^{26}$ and Klawans ${ }^{27}$ report a difference in the long-term response to levodopa based on sign. They observed that the drug-related improvement of tremor compared with all other signs measured appeared to be the best maintained during the course of the disease whereas improvement in gait clearly deteriorated. A specific influence (or lack of influence) of age, however, was not addressed in either report.

In our study, there was an indication that patients maintaining a longer benefit from a single dose of carbidopa and levodopa were generally those who had been diagnosed as having Parkinson's disease for a shorter period of time. This observation is consistent with the hypothesis that the duration of motor response to levodopa is dependent on the capacity of surviving nigrostriatal terminals to store newly synthesised dopamine. We note, however, that a comparison of the duration of the response to levodopa with the duration of the disease, as part of the multiple regression analysis, did not reach significance $(p=0.097)$. Thus our inability to find a strong association between the duration of disease and the duration of the response to levodopa is probably best explained by an influence of a factor or factors not addressed in this study. In this regard, Kempster et $a l^{28}$ have described peripheral levodopa pharmacokentics as an important determinant of the duration of the response in patients receiving oral carbidopa and levodopa.

We conclude that factors associated with ageing have a significant impact on one important aspect of levodopa efficacy: the magnitude of the response to the drug. It is suggested that changes due to ageing, for example, striatal receptor loss and additional cell loss in the nigra, become superimposed on Parkinsonian pathology resulting in additional disability which is not amenable to levodopa replacement. Relevant to this theory is the demonstration in individuals without Parkinson's disease that "normal" age-related extrapyramidal signs are not responsive to levodopa therapy. ${ }^{29}$

We gratefully acknowledge Dr Martin Albert for his review and suggestions in the preparation of this manuscript. We also thank Errol Baker for his help in the statistical analysis of our data. Supported in part by VA Merit Review Grant 001 and the Harold and Ellen Wald Parkinson's Disease Fund.
1 Hunter KR, Laurence DR, Shaw KM, Stern GM. Sustained levodopa therapy in parkinsonism. Lancet 1973; 2:929-31.

2 Marsden CD, Parkes JD. Success and problems of longterm levodopa therapy in Parkinson's disease. Lancet 1977;1:345-9.

3 Marsden CD. "On-off" phenomenon in Parkinson's disease. In: Rinne UK, Klinger M, Stamm G, eds. Parkinson's disease: current progress, problems and management Amsterdam: Elsevier, 1980:241-54.

4 Muenter MD. Should levodopa be started early or late? Can f Neurol Sci 1984;11:195-9.

5 Nutt JG. On-off phenomenon: relation to levodopa pharmacokentics and pharmacodynamics. Ann Neurol 1987;22: 535-40.

6 Mouradian MM, Juncos JL, Fabbrini G, Chase TN. Motor fluctuations in Parkinson's disease: pathogenetic and therapeutic studies. Ann Neurol 1987;22475-9.

7 Doller HJ, Connor JD. Changes in neostriatal dopamine concentrations in response to levodopa infusions. $f$ concentrations in response
Neurochem 1980;34:1264-9.

8 Spencer SE, Wooten GF. Altered pharmacokentics of L-dopa metabolism in rat striatum deprived of dopaminergic innervation. Neurology 1984;34:1105-8.

9 Granerus AK, Steg G, Svanborg A. Clinical analyses of factors influencing $L$-dopa treatment of Parkinson's syndrome. Acta Med Scand 1972;192:1-11.

10 Lieberman A, Dziatolowski M, Kupersmith M, et al. Dementia in Parkinson's disease. Ann Neurol 1979;6: 355-9.

11 Pederzoli M, Girotti F, Scigliano G, Aiello G, Carella F, Caraceni T. L-dopa long-term treatment in Parkinson's disease: age-related side effects. Neurology 1983;33: 1518-22.

12 Gauthier S, Gauthier L. Current status of levodopa therapy in idiopathic Parkinson's disease. Can $₹$ Neurol Sci in idiopathic Part

13 Diamond SG, Markham $\mathrm{CH}$, Hoehn MM, McDowell FH, Muenter MD. Effect of age at onset on progression and mortality in Parkinson's disease. Neurology 1989;39: $1187-90$.

14 Severson JA, Marcusson J, Winblad B, Finch CE. Agecorrelated loss of dopaminergic binding sites in human basal ganglia. $\mathcal{f}$ Neurochem 1982;39:1623-31.

15 Wong DF, Wagner HN, Dannals NE, et al. Effects of age on dopamine and serotonin receptors measured by positron tomography in living human brain. Science;226:1393-6.

16 Govoni S, Memo M, Saiani L, Spano PF, Trabucchi M. Impairment of brain neurotransmitter receptors in aged rats. Mech Ageing Dev 1980;12:39-46.

17 Marksman M, Ahn HS, Thal LJ, et al. Aging and monoMarksman M, Ahn HS, Thal LJ, et al. Aging
amine receptors. Fed Proc 1979;38:1922-6.

18 Memo M, Lucchi L, Spano PF, Trabucchi M. Aging process affects a single class of dopamine receptors. Brain Res 1980;202:488-92.

19 Leenders KL, Antonini A, Schwartz J, Oertel WH, Hess K, Weindl A. Striatal dopamine $\mathrm{D}_{2}$-receptors in "de novo" parkinsonian patients measured using $\mathrm{PET}$ and [ " $\mathrm{C}] \mathrm{ra}-$ clopride. Neurology 1992;42 Supp 3:295.

20 Durso R, Szabo G, Davoudi H, Feldman RG. Magnitude of response to levodopa in Parkinson's disease as it relates to peripheral and central measurements of levodopa and associated metabolites. Clin Neuropharm 1989;12: 384-92.

21 McGeer PL, McGeer EG, Suzuki JS. Aging and extrapyramidal function. Arch Neurol 1977;34:33-5.

22 Gibb WR, Lees AJ. Anatomy, pigmentation, ventral and dorsal subpopulations of the substantia nigra, and differential cell death in Parkinson's disease. $\mathcal{F}$ Neurol Neurosurg ential cell death in Parkinson's
Psychiatry 1991;54:388-96.

23 Goldberg A, Melamed E, Rosenthal J, Reches A, Tiqva P. Synthesis of dopamine from exogenous levodopa in striatum: effect of graded destruction of dopaminergic nerve terminals by increasing doses of MPTP. Neurology;40 (Suppl 1):291.

24 Burns RS, Kopin I, Weiss V, Roznoski M, Ebert M. Decreased LAAAD in striatum influences brain l-dopa metabolism in MPTP treated rhesus monkey with severe parkinsonism. Neurology 1991;41 (Suppl 1):378.

25 Gibb WR, Lees AJ. A comparison of clinical and pathological features of young- and old -onset Parkinson's disease. Neurology 1988;38:1402-6.

26 Bonnet AM, Loria Y, Saint-Hilaire M, Lhermitte F, Agid Y. Does long-term aggravation of Parkinson's disease result from nondopaminergic lesions? Neurology 1987;37: from n39-42.

27 Klawans HL. Individual manifestations of Parkinson's disease after ten or more years of levodopa. Movement Dis 1986;1:187-92.

28 Kempster PA, Frankel JP, Bovingdon M, Webster R, Lees AJ, Stern GM. Levodopa peripheral pharmacokinetics and duration of motor response in Parkinson's disease. $\boldsymbol{f}$ Neurol Neurosurg Psychiatry 1989;52:718- 23

29 Newman RP, LeWitt PA, Jaffe M, Calne DB, Larsen TA. Motor function in the normal ageing population: treatment with levodopa. Neurology 1985;35:571-3. 